\title{
Ultra-Luminous Sources in Nearby Galaxies
}

\author{
Richard Mushotzky \\ NASA Goddard Space Flight Center, Greenbelt, MD 20771, USA
}

\begin{abstract}
I briefly review much of the X-ray and optical data on the nature of the ULXs in nearby galaxies. I present new results on radio emission, finding that the radio is usually rather luminous and extended. I review the X-ray data on timing and spectra. There is no direct evidence in the X-ray data for either geometric or relativistic beaming and in 4 objects direct evidence against beaming. I argue that the X-ray timing and spectral properties of these objects, are in general, not good analogs of AGN or galactic black holes and that the ULX may represent a new mode of accretion only rarely seen in other objects.
\end{abstract}

\section{§1. Introduction}

Since their discovery in observations of nearby galaxies with the Einstein observatory (Fabbiano et al. 1989), the existence of a class of ultra-luminous X-ray sources has been well documented. For the purposes of this paper, we shall call a source "ultra-luminous" or a ULX, if it is not at the galaxy nucleus, it is spatially unresolved with Chandra or is time variable and if its observed bolometric luminosity exceeds the Eddington limit for a $20 M_{\odot}$ black hole $\left(2.8 \times 10^{39} \mathrm{ergs} / \mathrm{sec}\right)$. Because black holes of greater than $20 M_{\odot}$ are not expected from "normal" stellar evolution (even from very massive stars, Fryer and Kalogera 2001) and are clearly different from the "normal" population of black hole X-ray sources as seen in the Milky Way we believe that this is the appropriate luminosity to consider. These objects are moderately numerous since they are in $\sim 1 / 4$ of all galaxies (Colbert and Ptak 2002). They are of great interest since they represent a population of possible intermediate mass $\left(20-5,000 M_{\odot}\right)$ black holes $(\mathrm{IMBHs})$ or a stage in the evolution of binaries not seen in the Milky Way. The existence of such a population poses strong theoretical challenges to their creation and fueling (talk by King this workshop), while if they are not IMBHs their high total luminosity and unique spectral and timing properties pose strong challenges to physical models for their radiation mechanism (Ebisawa et al., these proceedings, Kubota et al., these proceedings). In defining the luminosity of these objects one must be careful to calculate the bolometric luminosity. For many of the models fit to the ULX X-ray spectra the $0.5-10$ $\mathrm{keV}$ observed band contains less than $40 \%$ of the total luminosity. Thus many of these objects are more luminous than reported in much of the literature.

As we will show in this paper many of the ULXs have properties not shared by AGN or galactic black holes and that, as a class, they show a wide variety of spectral and temporal behaviors which suggest that they may be several types of object and not represent a uniform population. 


\section{§2. Classes of models}

After the discovery of these objects many models were suggested to explain their origin. They basically fall into 3 classes: supernova remnants, non-isotropic emission from "normal" black hole binaries and "normal" emission from intermediate mass black holes.

\subsection{Supernovae in dense environments}

These objects are known to exist and can have observed luminosities of $L_{x} \sim$ $10^{38}-2 \times 10^{40} \mathrm{ergs} / \mathrm{sec}$ (e.g. SN 1995N, Immler and Lewin 2002). Their high luminosity is due to the rapid dissipation of the supernova shock energy in a dense environment and thus these objects are associated with historical supernova. Their luminosities are observed to decline monotonically over a few years (Immler and Lewin 2002). However, there exist anomalous SNR, such as the SNR in NGC4449, whose XMM X-ray spectrum are clearly thermal and are associated with optical objects which have classical SNR emissicon line ratios, and which have very high luminosities, but are not associated with historical remnants and whose luminosity does not decline much with time (Patnaude and Fesen 2003).

The other two possibilities are "theoretical" objects postulated to exist to produce the high observed luminosities of the ULX.

\subsection{Non-isotropic emission}

The high luminosities of the ULX may be explained by non-isotropic emission from X-ray binaries: either from a "normal" high-mass X-ray binaries (HMXB) or micro-blazars (beamed emission from relativistic jets). In both of these classes of models the observed high luminosity is due to either geometrical or relativistic beaming (Körding et al.), a short lived super-Eddington phase (King 2003) or a stable super-Eddington luminosity due to a geometrical arrangement of emission and absorption regions which allows the photons to escape without interacting with surrounding material (Begelman 2002). While micro-quasars certainly exist in the Milky Way (see Mirabel, these proceedings) the ULXs can be 1000 times more luminous and would be a rather different type of object.

\subsection{Accretion onto massive objects}

The third class of models invokes the existence of very massive objects in order to reproduce the bolometric luminosity of these objects with "normal" accretion processes. To not exceed the Eddington limit one needs a mass between $20-2000 M_{\odot}$ and thus this class of models posits the existence of intermediate-mass black holes (IMBHs). There also exist "lost" LLAGN which are low-luminosity AGN which are not detected by optical techniques and are not in the "obvious" nucleus of the galaxy (e.g. NGC3256 Neff et al. 2003). These objects are presumed to have been displaced from the apparent nucleus via a strong merger. Their relative lack of optical emission lines is not unusual amongst Chandra selected AGN (Mushotzky 2004). 


\section{$\S 3$. What is the available data?}

There is now a considerable body of data from archival Rosat (Colbert and Ptak 2002), Chandra and XMM data (Humphrey et al., Swartz et al., Ptak et al.) about the relative numbers, X-ray luminosity, luminosity function and X-ray colors of the ULXs in a reasonable number of nearby galaxies. There have been extensive searches for counterparts in other wavelength bands (optical, near-IR and radio). A reasonable number of objects have moderate to high quality X-ray CCD spectra from ASCA, Chandra and XMM in the $0.3-10 \mathrm{keV}$ band (Kubota et al., these proceedings, Ebisawa et al., these proceedings).

There is a large amount of data based on X-ray time variability on long (years) to short (seconds) time scales (e.g. Roberts and Warwick 2000) for the brighter sources, but with very uneven time sampling. There are extensive studies of the correlations of ULX properties with galaxy properties (Colbert et al. 2003, Gilfanov et al. 2003, Gilfanov, these proceedings, Swartz et al. 2003).

\section{$\S 4$. The critical issues}

The main issue in this field is, clearly, "what is the true nature of the ULX sources and what is their emission mechanism?" This boils down to whether $M>20 M_{\odot}$ objects really exist, and if they do not, how can one produce the observed very high luminosities.

\subsection{Arguments against $M>20 M_{\odot}$ objects}

As discussed in detail by King and co-workers (talk by King this workshop) there are several substantial arguments against $M>20 M_{\odot}$ objects being the origin of most of the ULXs. These arguments can be grouped into two parts.

\subsubsection{Astronomical}

There are severe difficulties in forming such massive objects and providing sufficient fuel to feed them. Considering their apparent high accretion rate $\left(10^{-8}\right.$ $\left.10^{-6} M_{\odot} / \mathrm{yr}\right)$ the lifetime of any companion must be short and thus, there must be many "quiescent" ULX for each luminous one.

If these objects have $M>20 M_{\odot}$ they cannot form from stellar evolution of single "normal" massive stars and their origin must lie in collective phenomena (Miller and Colbert 2003). It is difficult to form such objects in classical binary stellar evolutionary scenarios unless the companion (which provides the "fuel") is also massive and thus has a short lifetime. If the objects are not formed as binaries, they must "acquire" a stellar companion via capture, which maybe difficult. If their true luminosities are so high one has to consider possible ablation of a companion which should further shorten the life of the system.

\subsubsection{Statistical}

ULX tend to be associated with recent star formation regions and their total numbers are correlated with the star formation rate (Gilfanov et al. 2003). A small number have possible optical associations with luminous stars, indicating that 
they are associated with massive, short lived stellar objects. Some show transitions similar to that seen in galactic black holes indicating that they are similar objects. As discussed in detail by Gilfanov (these proceedings) the overall luminosity function of galaxies does not have a "feature" associated with the ULXs indicating that there is smooth transition between objects more and less luminous that a $20 M_{\odot}$ object radiating at the Eddington limit.

However in all models in which the ULXs are less than $20 M_{\odot}$ the observed luminosity must be due either to beaming in an intrinsically Eddington limited object, or via radiation from a super-Eddington object by some means (see Begelman 2002, King 2003 for such possibilities).

\subsection{Arguments against $M<20 M_{\odot}$ objects}

While such astronomical/theoretical arguments seem daunting there are many direct observations which seem to rule out beaming or super-Eddington luminosities in these objects.

What are the arguments against ULX being "normal" $M<20 M_{\odot}$ objects?

\subsubsection{Data}

The X-ray spectra of the ULX are often not like AGN or normal galactic black holes (see below) indicating that they are not simple analogs of these objects. The ULX can have state transitions like "normal" black holes (Kubota et al. 2001) or in the opposite sense from the vast majority of galactic objects (Fabbiano et al. 2003, Dewangan et al. 2004). Their bolometric luminosities can reach $1000 L_{\text {edd }}$ for a 1 solar mass object making beaming or super-Eddington models rather extreme.

There is direct evidence against beaming in several objects: viz the QPO in M82, the broad Fe lines in two ULX M82 (Strohmayer and Mushotzky 2003) and the Circinus galaxy (Weisskopf et al. 2003), and the evidence for eclipses in 2 objects (the Circinus galaxy ULX and one of the ULX in M51 (Liu et al. 2002)). At least one object has a break in the power density spectrum (PDS) at the frequency predicted for $M \sim 1000 M_{\odot}$ objects (Cropper et al. 2004).

As shown later in this paper many of the ULXs have associated, very luminous extended radio sources, very different from any associated with Milky Way black holes. There are a few ULXs with highly luminous photoionized nebulae around them (Pakull and Mironi 2002). If these nebulae are photoionized by the ULX, as indicated by their association and unusual optical spectra, one requires a high total ULX luminosity. There is also a general lack of stellar optical identifications; in many of the galaxies observed with both HST and Chandra the sensitivity threshold of HST is such that luminous O star counterparts would have been detected. While the ULXs are associated with star forming regions, they lie near but not in them. This requires, that if the ULX are created in these star forming regions, that the ULXs have a high space motion (Kaaret et al. 2004) but "carry around" the extended radio sources and optical nebulae, which to this author seems unlikely. Quite a few of the ULXS have "soft" components well fit by a low $k T$ black body whose temperature and flux are consistent with a high mass (Miller, these proceedings). 


\subsubsection{Theory}

From a theoretical point of view there are no known mechanisms for the required beaming $(>100)$ in the most luminous objects other than relativistic effects. The observed X-ray spectra and time variability behavior does not resemble that of known relativistically beamed objects (such as Bl Lacs). In many sources (e.g. NGC2276 Davis and Mushotzky 2004) the observed luminosity function lacks the large number of lower luminosity objects (Körding, these proceedings) required in a relativistic beaming scenario and thus is not consistent with beaming. The ULX are not "ultraluminous" in other wavelength bands like AGN are, thus placing strong constraints on models (King 2003) in which the actual luminosity is super-Eddington. While quasars and Seyfert galaxies radiating near the Eddington limit (Czerny et al. 2003) appear to have a "universal" broad band spectrum that is not seen in the ULXs, reducing the likelihood that these objects are radiating near the Eddington limit.

\section{$\S 5$. Where do they come from?}

If the ULX are really $M \sim 100 M_{\odot}$ objects, there are, at present, two possibilities for their origin. The ULX may be created in the early universe; detailed calculations of the first stars to form (e.g. Abel 2003) indicate that $M \sim 200-1000 M_{\odot}$ objects should be created, and that they are numerous and lie in regions that will later become galaxies.

Alternatively, the ULXs could be created in dense stellar regions (e.g. globular clusters Miller and Colbert 2003 or dense star clusters (McMillan and Portegies Zwart 2003)). However since most ULXs today are not observed to be in dense clusters this requires that either the ULXs are ejected or that the clusters dissolve or otherwise become invisible. If they are not supermassive objects then they must represent an unusual stage of stellar evolution and/or some new mode of accretion.

\section{$\S 6 . \quad H o w$ do we determine their nature?}

If ULXs are intermediate mass black holes they should have properties that scale from the better studied AGN at high mass and galactic black holes at low mass. These properties include the detailed nature of their time variability, the spectral form of their broad band X-ray spectra and the detailed spectra in Xray/radio/optical bands. In particular, one would like to measure properties that scale with mass such as the temperature of the low energy component, where one expects that $k T \sim M^{1 / 4}$, and the characteristic time scale of X-ray variability (Markowitz et al. 2003) where the break in the power density function scales with mass. As we show in this paper some of these scalings have been observed for some of the objects but most of the ULXs do not show the expected relationships. 


\section{$\S 7 . \quad$ What can we learn from optical associations?}

Much progress in understanding the nature of both galactic and extragalactic X-ray sources has come from detailed optical "follow-up" observations and the original hope was that similar programs, that succeeded so well in identifying the nature of galactic and extragalactic X-ray sources, would be the key to understanding the ULXs. Unfortunately this has turned out to be considerably harder than first hoped. If unique "identification" of optical counterparts is obtained one could estimate the masses of ULXs, their evolutionary history and discriminate between models. If optical emission line nebulae are associated with the ULX they can be used as calorimeters to derive true isotropic luminosities of the objects, constrain the lifetime of the ULXs, the form of the ionizing continuum and place them in their evolutionary context.

\subsection{Optical nebulae}

Most of the reported optical nebulae which are associated with the ULXs (Pakull and Mironi 2003) are very big 200-600 pc, and very energetic, with kinetic energies $\sim 10^{52}-10^{53} \mathrm{ergs} / \mathrm{sec}$ much more than standard SNR. There are at least 2 variable ULXs associated with nebulae that were thought to be supernova remnants, based on the nature of the optical emission lines (Roberts et al. 2003a and b). The detailed

optical spectra of these nebulae can, in principle, distinguish shock vs photoionization origins for the optical emission lines and determine whether they may be excited by the "central" X-ray source. Many of the nebulae are rather unusual, having both strong [OIII], NeIII and HeII emission lines as well as lower ionization lines of [OI] and [SII]. The most interesting case so far is the nebulae associated with the ULX in the dwarf galaxy Holmberg II (Pakull and Mironi 2003) which shows strong HeII 4686 and [OI] 6300. Since HeII is produced by recombination it requires a strong high energy source of photoionization with an isotropic luminosity of greater than $3 \times 10^{39} \mathrm{ergs} / \mathrm{sec}$ to produce the observed optical emission line spectrum.

Over 4 ULX are inside bubble-like nebulae, 200-400 pc in size, much larger than SNR in the Milky Way. It is not yet clear if the nebulae associated with the ULXs are highly unusual and/or how statistically likely a chance coincidence between such a large and luminous nebula with a random ULX would be.

\subsection{Optical stars}

Even with the precision Chandra positions there is often no unique optical counterpart to the ULX (Cropper et al. 2004). While several counterparts have been reported from ground based observations, at least 2 of them "break up" in deep HST images (Goad et al. 2002, Cropper et al. 2004) or are extended (Immler et al. 2003) indicating that much of the optical flux comes from several objects or is a star cluster. This variety can be seen in NGC4559, as is seen clearly in the HST images (Cropper et al. 2004) the southern source (X-7) has 5 visible counterparts inside the Chandra error circle, while in the field of the northern ULX (X-10) there are no counterparts. At present there are several other ULX with possible optical counterparts, 2 with a probable O-star counterpart (Liu et al. 2002b, Goad et al. 
2003), one with a globular cluster in a spiral galaxy (Wu et al. 2002) and several with globular clusters in elliptical galaxies (Angelini et al. 2001, Maccarone et al. 2003).

However the sensitivity of HST is such that only the most luminous stars can be recognized at $D<15 \mathrm{Mpc}$. There has not yet been statistical work on the likelihood that the counterparts are real. While the random association of a luminous $\mathrm{O}$ star with a ULX is unlikely, the fact that ULX are often near star forming regions raises the random probability considerably. As opposed to the early searches for galactic and active galaxy counterparts to X-ray sources, none of the ULX counterparts show "unusual" optical colors or variability. This considerably increases the difficulty of directly associating the optical counterpart with the ULX.

The optical studies have clearly shown that the ULXs have very high X-ray to optical ratios. X-ray selected AGN from the Rosat all sky survey tend to have $\log F(x) / F$ (opt) 1, which for the brighter ULXs with $F(x) \sim 10^{-12} \mathrm{ergs} / \mathrm{cm}^{2} / \mathrm{sec}$ in the $0.5-10 \mathrm{keV}$ band would have optical counterparts at the $16 \mathrm{mag}$ level or 6-9 magnitudes brighter than usually observed. Thus the ULXs do not have the optical properties expected if they were simple extensions of AGN.

However low mass X-ray binaries in the Milky Way have $F(x) / F($ opt $) \sim 100-10^{4}$ consistent with the observed values in the ULXs. When these objects are luminous most of the observed light comes from the accretion disk. If the detected ULXs companions are high mass stars, the observed optical light is consistent with that expected from a massive companion star with little or no contribution from an accretion disk. The present data place only weak constraints on the optical emission from an accretion disk scaling from X-ray binaries in Milky Way.

\section{$\S 8$. Radio observations of ULXs}

In the search for counterparts in other wavelength ranges it is natural to look in the radio band. The radio data have excellent angular resolution and accuracy for help in finding an optical counterpart and contain unique diagnostics for the nature of the source (AGN, SNR, beaming, HII region, etc.). Also the high sensitivity of the VLA allows the detection of intrinsically low luminosity emission. We (S. Neff, N. Miller, RM) have cross correlated the FIRST and NVSS radio catalogs with Chandra/XMM for nearby galaxies. This allows a quick search for possible radio counterparts for further detailed VLA analysis. So far we have $>12$ "hits" (with an angular distance $\delta \theta<1.5^{\prime \prime}$ ) between FIRST radio sources and non-nuclear X-ray sources (we also have quite a few with NVSS and XMM data with larger $\delta \theta$ ) and several have "good" VLA data.

Because of the requirement that all sources be either in the FIRST or NVSS surveys their fluxes are greater than 1-2 mJy, or somewhat larger if the host galaxy has bright diffuse galactic radio emission. A quick analysis shows that, the ULX have a radio/X-ray ratio less than for Bl Lacs in general (Rector et al. 2002, LaurentMulhlensin et al. 1998) and that the radio/optical ratio is larger; a typical Bl Lac optical to radio spectral index of 0.5 for a source at a radio flux of 1 mJy would predict an optical flux of $\sim 22-23 \mathrm{mag}$, which is brighter than or equal to most of the 
sources. The observed optical-X-ray ratios of the ULX are not consistent with that of most $\mathrm{Bl}$ Lac objects; that is the ULXs do not fall into the classification regions for $\mathrm{Bl}$ Lacs in the X-ray-optical-radio plane. This lessens the likelihood that the ULX resemble these beamed objects.

A major surprise from our result is that a significant fraction of the radio counterparts to the ULX sources are resolved by the VLA. We had anticipated that the radio emission would be point-like based on the original discovery by Kaaret et al. (2003) of a radio counterpart to the ULX in NGC5408. Kaaret et al. claim that the radio counterpart to the ULX is compact and thus would be consistent with the radio emission being due to relativistic beaming in the ULX. The relative lack of point-like radio counterparts in our mini-survey is a strong argument against relativistic beaming models. In Table I we show the radio properties of several of the ULX sources. Columns 3, 4 and 5 are the luminosity of the radio structures in units of Cas-A (the most luminous SNR in the Milkyway), the radio power in watts and the radio spectral index.

A simple calculation shows that the sensitivity of FIRST limits all the radio counterparts, at distances greater than $3 \mathrm{Mpc}$, to be more than 3 times as luminous as the most luminous radio source in the Milky Way, the supernova remnant Cas-A, and that to be resolved by the VLA the objects have to be rather large $(>20 \mathrm{pc}$ at $3 \mathrm{Mpc}$ distance). Thus their mere detection and resolution show that these objects are very luminous and very large for SNR or HII regions. Our results have been confirmed by Wang et al. 2003 who find several large and luminous resolved radio counterparts in NGC 3556, which because it is not a FIRST source, did not enter our sample.

The morphologies of the radio sources vary (Fig. 1) but some look distinctly like double radio sources. Their maximal cooling times (if emission is thermal like in HII regions) is $<3 \times 10^{8}$ yrs if there is no continuous energy injection and an order of magnitude shorter if they are synchrotron sources in equipartion. So far only one source has clear nature, the SNR in NGC4449 (which at $D=3 \mathrm{Mpc}$ has a

Table I. ULXs.

\begin{tabular}{|c|c|c|c|c|c|c|}
\hline NAME & $\begin{array}{c}\text { Distance } \\
(\mathrm{Mpc})\end{array}$ & Size $(p c)$ & \# Cas-A's & Radio Power & $\begin{array}{l}\text { Spectral } \\
\text { index }\end{array}$ & Notes \\
\hline NGC2782 & & & 2800 & $1.5 \mathrm{E} 21$ & & 3 peaks \\
\hline Starburst & 34 & 80 & 1100 & $6 \mathrm{E} 20$ & $\sim-0.3$ & Resolved arc \\
\hline galaxy & & & 600 & $3 \mathrm{E} 20$ & & of emission \\
\hline NGC3877 & 12 & $260 \times 170$ & 85 & $4 \mathrm{e} 19$ & -0.1 & $\begin{array}{c}\sim 7^{\prime \prime} \text { from } \\
\text { nucleus-jet? }\end{array}$ \\
\hline NGC4314 & 13 & $<125$ & 20 (each) & $1 \mathrm{E} 19(2)$ & -0.4 & 2 parts \\
\hline NGC4449 & 2.8 & $8 \times 4$ & $\sim 10$ & $5 \mathrm{E} 18$ & Steep, 1.7 & SNR \\
\hline NGC4490 & 6.6 & $\begin{array}{c}13 \text { (core) } \\
\sim 65\end{array}$ & $\sim 6$ & $3 \mathrm{E} 18$ & -0.5 & $\begin{array}{c}\text { Core } \\
\text { halo/double }\end{array}$ \\
\hline HoII & 2 & $40 \times 30$ & $\sim 1$ & $5 \mathrm{E} 17$ & -0.3 & \\
\hline NGC3256 & & $90 \times 270$ & $\begin{array}{c}1000 \\
100 \\
\end{array}$ & & -0.8 (ULX) & $\begin{array}{l}\text { Twin AGN+ } \\
\text { ULX }\end{array}$ \\
\hline NGC5408 & 4.8 & & 1.5 & & $>-1$ & Kaaret et al. \\
\hline
\end{tabular}


radio luminosity of $10 \times$ Cas-A). While young SNR can be as luminous as $L(x)>10^{3}$ Cas-A, this high a luminosity in an "old" SNR is highly unexpected.

Some examples of the radio emission are NGC4631 :

We see the general association of radio emission and ULXs. The most luminous radio/X-ray source has $\log L(x) \sim 39.7(.3-10 \mathrm{keV})$ and can be well fit by a disk black body model with $k T$ diskbb $=1.2 \mathrm{keV}$, and column density $N(H)=2.6 \times 10^{22}$. This object is located in the same place as the CO wind discovered by Rand (1999). Rand argues that the presence of the $\mathrm{CO}$ wind required roughly $10^{54}$ ergs of kinetic energy, considerably more than can be provided by a single supernova. The brightest X-ray source in the galaxy (to the west - not in the image in Fig. 1) has $F(x)=2.6 \times 10^{-12}$ and a $L_{\text {bol }} \sim 3.8 \times 10^{40} \mathrm{ergs} / \mathrm{sec}$ and is well fit by a simple power law with column density $N(H)=3.4 \times 10^{21}$.

Holmberg II (UGC4305):

This dwarf galaxy contains a very luminous source with $L_{\mathrm{bol}} \gtrsim 2 \times 10^{40} \mathrm{ergs} / \mathrm{sec}$ (Dewangan et al. 2004). The VLA source is coincident with the Chandra source $\left( \pm 0.5^{\prime \prime}\right)$ and is resolved, with an effective size of $2 \times 1.4^{\prime \prime}$ at $4.86 \mathrm{Ghz}$ and smaller at $1.4 \mathrm{Ghz}$ (for an equivalent size of $\sim 20 \times 25 \mathrm{pc}$ ). Its flat radio spectral index $-0.29 \pm 0.35$, is unusual for a thermal supernova remnant and the NVSS flux of $15 \mathrm{mJy}$ corresponds to a radio luminosity of $12 \times$ Cas-A, while the VLA resolved flux is $\sim 0.1$ of this, consistent with the observed morphology.

The XMM spectrum of the ULX shows a strong soft component (cf. Dewangan et al. 2004) which can be well fit by a diskbb model. The presence of a strong black body component, for this luminous ULX, inside a bright extended radio source is quite inconsistent with beaming in our line of sight! One should also note that the X-ray spectrum of the radio emitting ULX in NGC5408 also requires a similar black body component (see below).

NGC4314:

This object has one of the most spectacular examples of a ring like optical emission line structure as seen by HST and a similar shaped radio structure surrounding the nucleus. The observed radio luminosity is too large in the "knots" to be simply the sum of a "reasonable" number of SN . The sources associated with the radio emission are X-ray sources X-1 and X-3 and have X-ray luminosities of $L(x) \sim 3 \times 10^{39}$ ergs/sec and $L(x) \sim 7 \times 10^{38} \mathrm{ergs} / \mathrm{sec}$ respectively.

NGC3877:

For this galaxy at $D=17 \mathrm{Mpc}\left(1^{\prime \prime}=83 \mathrm{pc}\right)$ the VLA source is exactly coincident with the Chandra source $\left( \pm 0.5^{\prime \prime}\right)$. The radio source has a flux of $\sim 3 \mathrm{mJy}$ and is resolved with a rough size of $2 \times .4^{\prime \prime}$ at $4.86 \mathrm{Ghz}$ and smaller at $1.4 \mathrm{Ghz}$ with a flat spectral index of $-0.13 \pm 0.35$. This translates to a size of $150 \times 300 \mathrm{pc}$ and a luminosity of $80 \times$ Cas-A. The object is only $\sim 7^{\prime \prime}$ away from optical nucleus and has 5 Chandra observations with an average luminosity of $L(x) \sim 6 \times 10^{38}$. There is nothing obvious in the HST images indicating that the radio emission is not due to a giant HII region.

NGC4490:

For this galaxy at $D=8 \mathrm{Mpc} 1^{\prime \prime}=39 \mathrm{pc}$ and the VLA radio image is coincident 

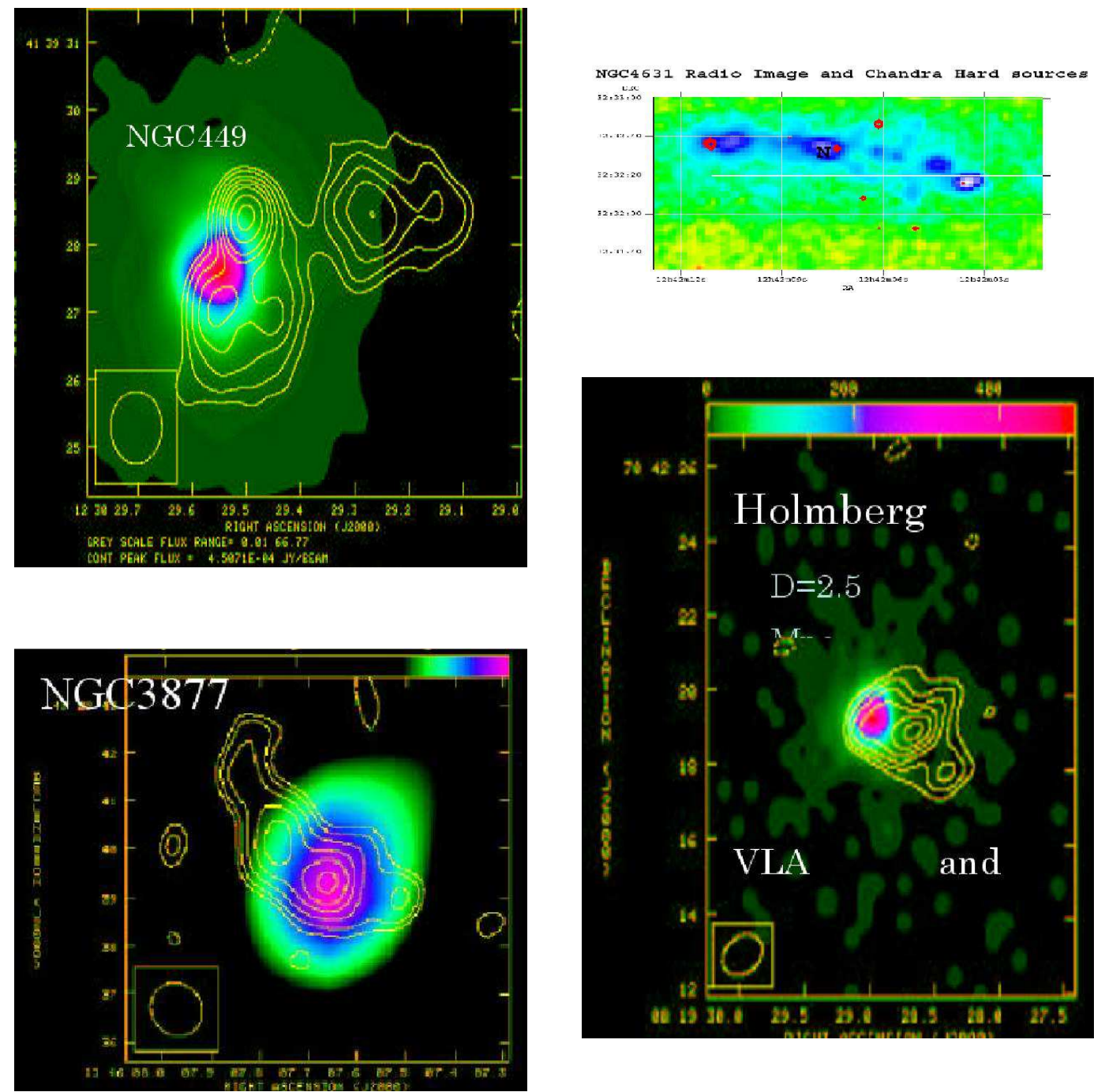

Fig. 1. The VLA radio images of 4 ULXs. The radio data are shown as contours superimposed on the grey scale Chandra image.

with the Chandra source $\left( \pm 0.5^{\prime \prime}\right)$. The source is resolved with a size of $2 \times .4^{\prime \prime}$ at $4.86 \mathrm{Ghz}$ about $75 \times 150 \mathrm{pc}$ and the spectral index is flat $-0.13 \pm 0.35$. With a flux of $\sim 3 \mathrm{mJy}$ it is $15 \times$ Cas-A. The X-ray flux varies between Chandra and XMM epochs with the Chandra $L(x) \sim 8 \times 10^{38}$.

\section{$\S 9 . \quad$ Nature of host galaxy}

ULXs can occur in any galaxy but are most frequent in rapidly star forming galaxies. However they can also occur in dwarfs, such as Holmberg II, and in elliptical galaxies with little present day star formation and in regions far from any obvious 
star formation (e.g. NGC1313 X-2 and NGC4559 X-10). In elliptical galaxies it seems as if the maximal luminosity is $10^{40} \mathrm{ergs} / \mathrm{sec}$, below the maximal luminosities seen in actively star forming galaxies. In NGC720, a nearby giant elliptical with no star formation, the number of ULXs is comparable to that of active star forming galaxies (Jeltema et al. 2003) and at least one is in a globular cluster. ULXs in globular clusters in elliptical galaxies are the most challenging to the association with star formation since these are old systems with no star formation in several gigayears.

In NGC4649, a nearby giant elliptical with no recent star formation ULX 69 (Colbert and Ptak 2002) has a good XMM spectrum. It is well fit by a power law (a diskbb is ruled out for the hard component) with an indication of a black body component and a bolometric luminosity of $\sim 10^{40} \mathrm{ergs} / \mathrm{sec}$. If the black body is physical it implies a size of $6 \times 10^{3} \mathrm{~km}$ which gives mass of $\sim 1000 M$ if the object is in the galaxy. There is a counterpart on the XMM OM UV data and the POSS plates and this possible ULX is either in a globular cluster or, alternatively, it is a background AGN only $3^{\prime}$ from a galaxy center. This raises the possibility that some of the ULX are projections of background AGN against the host galaxy. Based on the observed ratio of X-ray to optical fluxes for AGN this is only likely for the optically brighter ULX counterparts, as has been recently noted to one of the sources near NGC720 (Arp et al. 2004).

\section{$\S 10 . \quad$ Time variability}

Time variability is frequently observed in the ULXs, arguing that most of them are single compact objects, rather than a sum of numerous lower luminosity objects in the same object. Because in the pre-Chandra/XMM era the count rates were usually low, most of the time variability data is on timescales between observations ranging from months to years (Roberts et al. 2002).

Some of the ULXs may show X-ray periods, with data being published for IC 342; 31 or 41 hrs (Sugiho et al. 2001), the Circinus galaxy ULX with 7.5 hrs (Weisskopf et al. 2003) and M51 X-1; with a $2.1 \mathrm{hr}$ period (Liu et al. 2002). The periods in the Circinus galaxy and M51 objects appear to be due to eclipses, indicating that the companion is a low mass star.

While most ULXs vary, many show low amplitude variability on long time scales which is very different than galactic black holes or Seyfert galaxies (except for the black hole candidate LMC X-1 !) (Fig. 2). It is not clear if the apparent low level of variability is due to the sporadic nature of the observations or is a true property of the objects - this can only be answered by denser sampling of the time series. There are also quite a few examples of objects with large amplitude variability (e.g Strickland et al. 2001) including a few "transients".

Many galactic black holes exhibit "quasi-periodic oscillations" (QPOs) which are clearly associated with the accretion disk and represent characteristic length scales close to the black hole. If the QPO frequency is associated with the Kepler frequency at the innermost circular orbit for a Schwarzschild black hole, then the frequency of the observed QPO in M82 (Strohmayer and Mushotzky 2003) of $0.06 \mathrm{~Hz}$ translates 

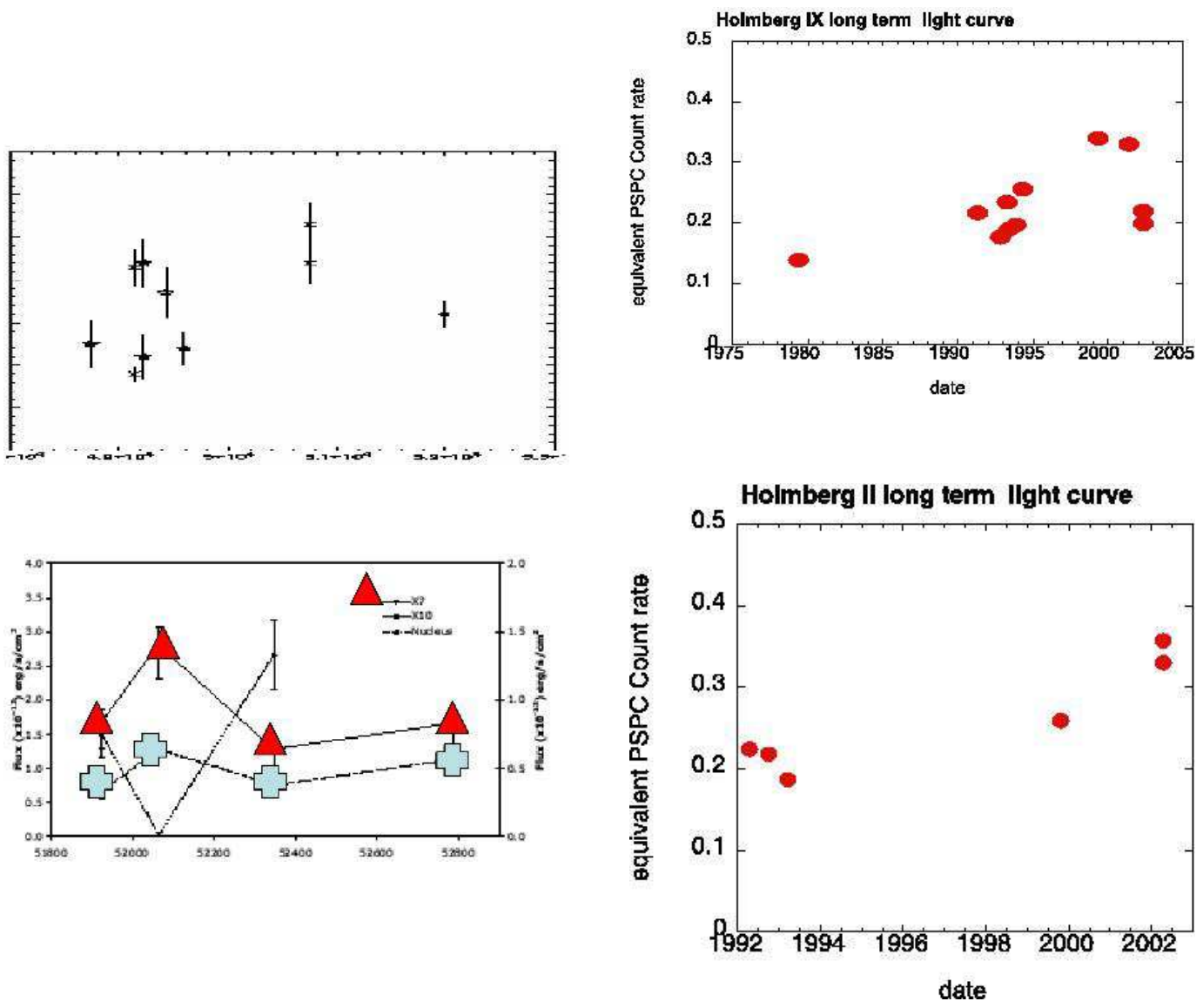

Fig. 2. The long time scale variability of 5 ULX; M81 X-9, Holmberg II, NGC2276 and the two ULX in NGC 4559.

to an upper limit on the mass of $1.9 \times 10^{4} M_{\odot}$, consistent with observed luminosity and an efficiency of $\sim 0.1$, similar to that of most AGN and galactic black holes.

It has long been known that galactic black holes exhibit characteristic power density spectra (PDS), flat at low frequencies and steep at high frequencies and that, in some sense, this is a defining character of these objects. The PDS for AGN shows a similar form, with the break frequency scaling as the mass of the object over a factor of $10^{7}$ in mass (Markowitz et al. 2003). Only XMM has the signal to noise to measure an accurate PDS for $\sim 5-10$ ULXs if their PDS scales from Cyg X-1 or Seyfert galaxies. The PDS for several XMM sources are well sampled, with good signal to noise. Preliminary analysis for several ULXs shows that many of them have low overall power (Fig. 3) with no more QPOs (yet) and thus the ULX, in general, do not have the "characteristic" BH power spectra. However there is a singular exception, the ULX X-7 in NGC4559 (Cropper et al. 2004). X-7 has a "classical" Cyg X-1 power spectrum, flat at low frequencies and steep at high and with an RMS variability of $37 \%$ it is very similar to Cyg X-1. However its break frequency of $\sim 28 \mathrm{mHz}$ is 50 times longer. Scaling that break frequency to mass (as 


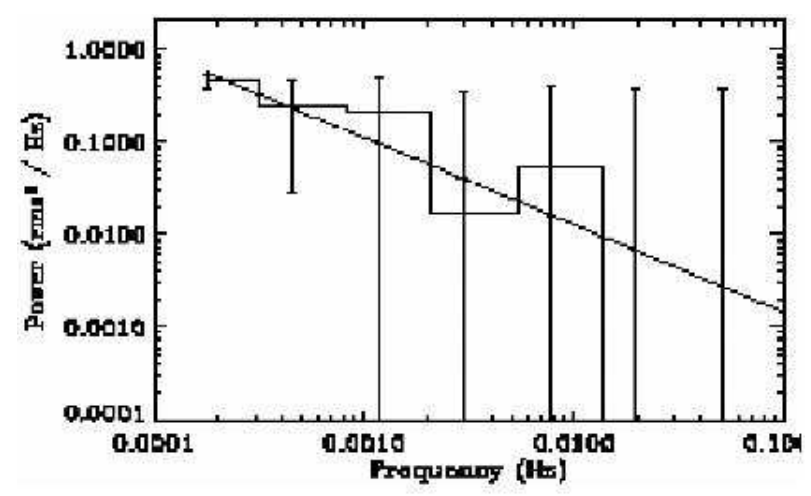

\section{M33 PDS- very little power at all timescales sampled}

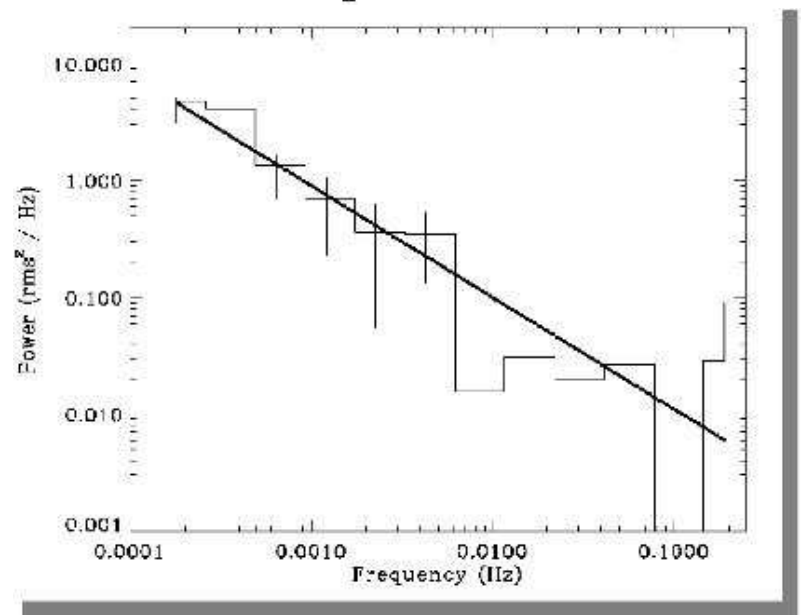

Fig. 3. The PDS for the central source in M33 and the Circinus ULX (Strohmayer p.c.) showing the low overall power in these objects and the absence of QPOs and a break in the PDS.

for AGN and Cyg X-1) gives $M \sim 10^{3} M_{\odot}$. In the same galaxy the other ULX, X-10, has a steep power law PDS with little high frequency power and no characteristic frequency.

We believe that the search for characteristic frequencies is, at present, the most productive way of determining the nature of the ULX. However to utilize this technique requires moderately long exposures of sources with $>1 \mathrm{ct} / \mathrm{sec}$. Since all black holes seem to show the same characteristic PDS a search for the characteristic frequency is crucial for a large sample. If it is indeed true that the form of the PDS is universal, then the absence of a low frequency break in several of the measured PDS implies rather large masses. 


\section{$\S 11 . \quad \mathrm{X}$-ray spectra}

To first order the X-ray spectra of accreting black holes can be modeled, for most AGN and galactic black holes by two forms, a pure power law and a power law with a disk black body component. If the X-ray spectrum of the ULXs can also be well described by this model, then a simple relation exists between the color temperature and luminosity of the disk black body component and the mass of the object is (Ebisawa et al. 2002) $T_{\text {color }} \sim 1.3 \mathrm{keV}\left(\left(T_{\text {color }} / T_{\text {eff }}\right) / 1.7\right)\left(M / M_{\text {edd }}\right)^{1 / 4}(M / 7 M)^{-1 / 4}$ where $T_{\text {color }}$ is the effective diskbb temperature fitted to X-ray spectra. Since the implied masses of the ULXs are greater than $20 M$, one expects $T_{\text {color }}<1 \mathrm{keV}$. However, based on ASCA data (Colbert and Mushotzky 1999, Makishima et al. 2000) many of the ULX have $T_{\text {color }}>2 \mathrm{keV}$ inconsistent with high masses.

Detailed calculations (Ebisawa et al., these proceedings) indicate that this "color temperature" problem is not generally solved in a Kerr metric. Thus the ULXs are too "hot" for their inferred "Eddington limited" mass and either the spectral model, masses or interpretation is wrong. It is clear that the ASCA data were not very "wrong" since high S/N XMM data confirm that some sources have curving spectra. However the spectral fits are not unique and these spectra can be fitted either by the diskbb models or by Comptonization spectra, as originally pointed out 15 years ago for LMXB spectra (White et al. 1988). For example in the case of M81 X-9, the XMM high signal to noise data are equally well fit by power laws with black body spectrum, Comptonization models and diskbb + power law, while for Holmberg II the BMC models of Shrader and Titarchuk, Compotonization and black body spectra and power law fit well while the diskbb with a black body soft component is a poorer fit. If the spectra can be interpreted as being due to Comptonization, as for low mass X-ray binaries, this alleviates the problems with high $k T$ disk black body models (see Kubuota, these proceedings). The very high signal to noise M33 X-1 spectrum (Fig. 4) can be very well fit by a Comptonized spectral form with low temperature and high optical depth. Only higher energy data can test which, if any, of these models are the correct description.

Given the uncertainties in the form of the continuum, factors of 2-3 uncertainty in the bolometric correction exist; in particular for fits with a steep power law the bolometric luminosity diverges at low energies. Since the optical fluxes are so low we know that the power law form cannot continue to arbitrarily low energies, contrary to the case in AGN.

With XMM quite a few sources require soft components which can be well fit by black body spectra with $0.1<k T<0.3 \mathrm{keV}$. The low temperature of the soft components is hard to detect with ASCA and Chandra ACIS spectra because of their relatively poor soft response. The exact value of $k T$ depends on the model used for the hard component and the form of the low energy absorption. In some cases, but not most of them, the adoption of a low abundance absorber removes the need for a soft component. However not all luminous sources require soft component. It is interesting that the range of inferred black body temperatures from these fits 0.1-0.2 $\mathrm{keV}$ is very similar to what is found for narrow line Seyfert galaxies, despite their apparent $10^{4}$ difference in mass. 

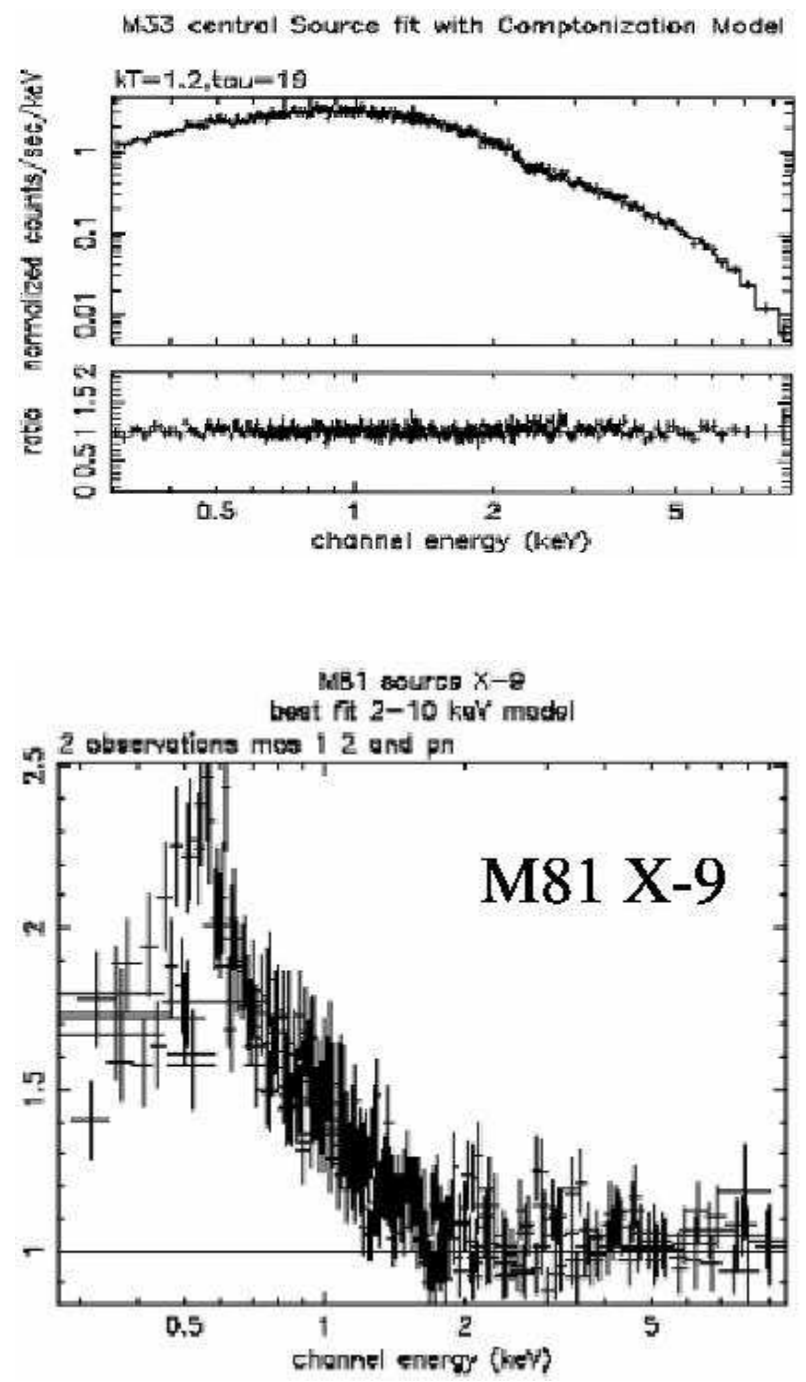

Fig. 4. Spectral fits to XMM data for several high signal to noise observations. (a) The fit to M33 central source with the Sunyaev-Titarchuk comptonization models with $k T \sim 1.22$ and $\tau \sim 10$. While this is an excellent fit to the data, the fitted parameters are outside the formal range of allowed values for the parameters. (b) The ratio of the data to a power law for the ULX X-9 in M81, illustrating the need for a strong soft component.

As an example of a detailed spectral fit, we consider the spectrum of X-7 in NGC4559 (Cropper et al. 2004). The XMM spectra have $\sim 20,000$ counts and are well fit by a power law $(\Gamma=2.23)$ and "black body" like component of $k T \sim 0.14$ $\mathrm{keV}$. The luminosity and temperature in the BB component give an effective size of $R \sim 3 \times 10^{9} \mathrm{~cm}$, which for the King and Pounds (2003) wind model gives a model mass $M \sim 2 \times 10^{3} M_{\odot}$, while the radius of a disk black body model is $R_{\text {diskbb }}=1.2 \times 10^{9}$ $\mathrm{cm}$; if this corresponds to $6 R_{G}$ then the black hole mass is $M \sim 1.6 \times 10^{3} M_{\odot}$. The BMC model (Titarchuk and Shrader 1999) gives a similar mass. Using these fits one derives a bolometric luminosity of $L_{\mathrm{bol}} \sim 6 \times 10^{40} \mathrm{ergs} / \mathrm{sec}$ or $L \sim 0.1 L_{\mathrm{Edd}}$ for the 
masses estimated from the spectral fits and the break in the power density spectra. There is no Fe $\mathrm{K}$ line, with an upper limit on the $E W<100 \mathrm{eV}$ for a narrow line and $200 \mathrm{eV}$ for a broad line.

Fe K lines:

The XMM data for bright sources with good $S / N$ typically do not show Fe lines, with the sole exception of M82 and the Circinus dipper. For the best spectra the upper limits are $\sim 50 \mathrm{eV}$; for several of the brigher object the upper limits are $<100$ $\mathrm{eV}$ for narrow lines. These limits are already interesting when compared to similar quality ASCA data for many Seyfert I galaxies. There are strong hints of oxygen lines in several sources but it is not clear if it is diffuse in origin or related to the ULX itself (e.g. Dewanag et al. 2004). For M82 and Circinus dipper the Fe K line is complex and broad. The EWs are $>100 \mathrm{eV}$; (in Circinus 2 lines of $\sim 180$ and 320 $\mathrm{eV}$ EW, in M82 the limits are $>130 \mathrm{eV}$ EW for a broad Gaussian and $\sim 250 \mathrm{eV}$ for a diskline.

The existence of strong and/or broad Fe K lines shows that, in these two objects, the continuum is not beamed since in order to produce lines of this strength requires the interaction of the X-ray continuum and a large solid angle of cold material, which is not available in the beaming scenarios.

\section{$\S 12$. Conclusion}

There is no direct evidence for beaming in any of the ULX: that is, they do not have the timing behavior, X-ray spectra or the broad band radio/X-ray-optical spectra characteristic of known types of beamed sources. In 4 sources there is direct evidence against beaming (M82 has both a QPO and a broad Fe line, NGC 4559 X-7 has a Cyg X-1 PDS, 2 sources are eclipsing (M51 and Circinus) and 2 (M82 and Circinus) have broad Fe lines). It is also becoming clear that many sources have a luminous soft black body like component. If this is due to an optically thick accretion disk (as is often assumed) it provides indirect evidence against beaming.

There is evidence for high intrinsic luminosity in several objects from the ionization of the associated optical nebulae and the presence of black body components. Some of the X-ray spectra show significant high energy curvature, different from that of known AGN and almost all of the known galactic black hole spectra, but similar to, but hotter, than that of LMXBs. Similar to LMXB most of the ULX do not have strong Fe K lines. Most of the X-ray PDS do not resemble that of Cyg X-1 or AGN with significantly less power over the frequency ranges sampled and only one source shows a PDS form of AGN or galactic black holes. There are associated luminous, large radio sources whose origin is not clear; this represents a new "type" of object associated with the ULXs. While their direct physical association has not yet been established, we are unaware of any such radio sources not associated with ULXs.

Thus the ULXs do not "look like" scaled up GBHCs or scaled down AGN nor like beamed versions of either one. The sum of the results do not "hang together". Either we are dealing with 3 or more "new" types of objects or we have to re-think what a black hole should "look like". I suspect that what we are detecting in many of these objects is Comptonized optically thick spectra, of relatively low temperature rather 
than the low optical depth, high temperature spectra characteristic of most AGN and galactic black holes in the low state. These are very exciting times for ULX research and I fully expect that there will be remarkable changes in our understanding of these objects in the next few years.

\section{Acknowledgements}

I would like to thank my collaborators at GSFC, Todd Strohmayer, Neal Miller and Susan Neff for major contributions to this work, and my NGC4559 collaborators, especially Mark Cropper and Roberto Soria for communication of results prior to publication. I would also like to thank the organizers for a most exciting meeting, which allowed many different ideas to be brought forth. I would also like to thank Professors K. Koyama and S. Mineshige for a beautiful introduction to Kyoto.

\section{References}

1) T. Abel, XEUS - studying the evolution of the hot universe, held at MPE Garching, March 11-13, 2002, ed. G. Hasinger, Th. Boller and A. N. Parmer, MPE Report 281 (2003), p. 1.

2) L. Angelini, M. Loewenstein and R. F. Mushotzky, Astrophys. J. 557 (2001), L35.

3) M. C. Begelman, Astrophys. J. 568 (2002), L97.

4) E. Colbert and A. Ptak, Astrophys. J. Suppl. 143 (2002), 25.

5) E. Colbert, T. Heckman, A. Ptak, D. Strickland and K. Weaver, astro-ph/0305476

6) M. Cropper, R. Soria, R. F. Mushotzky, K. Wu, C. B. Markwardt and M. Pakull, astro-ph/0311302

7) B. Czerny, M. Nikolajuk, A. Rózanska, A.-M. Dumont, Z. Loska and P. T. Zycki, Astron. Astrophys. 412 (2003), 317.

8) G. C. Dewangan, T. Miyaji, R. E. Griffiths and I. Lehmann, astro-ph/0401223

9) K. Ebisawa, P. Zycki, A. Kubota, T. Mizuno and K. Watarai, Prog. Theor. Phys. Suppl. No. 155 (2004), 67.

10) G. Fabbiano, Annu. Rev. Astron. Astrophys. 27 (1989), 87.

11) G. Fabbiano, A. Zezas, A. R. King, T. J. Ponman, A. Rots and F. Schweizer, Astrophys. J. 584 (2003), L5.

12) C. L. Fryer and V. Kalogera, Astrophys. J. 554 (2001), 548.

13) M. Gilfanov, H. -J. Grimm and R. Sunyaev, astro-ph/0309725

14) M. R. Goad, T. P. Roberts, C. Knigge and P. Lira, Mon. Not. R. Astron. Soc. 335 (2002), L67.

15) P. J. Humphrey, G. Fabbiano, M. Elvis, M. J. Church and M. Balucinska-Church, Mon. Not. R. Astron. Soc. 344 (2003), 134.

16) S. Immler and H. G. Lewin, in Supernovae and Gamma-Ray Bursts, ed. K. W. Weiler (Springer, New York, 2002); astro-ph/0202231

17) S. Immler, Q. D. Wang, D. C. Leonard and E. M. Schlegel, Astrophys. J. 595 (2003), 727.

18) P. Kaaret, S. Corbel, A. H. Prestwich and A. Zezas, Science 299 (2003), 365.

$19)$ P. Kaaret, A. Alonso-Herrero, J. S. Gallagher, G. Fabbiano, A. Zezas and M. J. Rieke, astro-ph/0312568

20) A. R. King, talk at this workshop.

21) A. R. King and K. A. Pounds, Mon. Not. R. Astron. Soc. 345 (2003), 657.

22) E. Körding, H. Falcke and S. Markoff, Astron. Astrophys. 382 (2002), L13.

23) A. Kubota, K. Makishima and C. Done, Prog. Theor. Phys. Suppl. No. 155 (2004), 19.

24) A. Kubota, T. Mizuno, K. Makishima, Y. Fukazawa, J. Kotoku, T. Ohnishi and M. Tashiro, Astrophys. J. 547 (2001), L119.

25) S. A. Laurent-Muehleisen et al., Astrophys. J. Suppl. 118 (1998), 127.

26) J.-F. Liu, J. N. Bregman, J. Irwin and P. Seitzer, Astrophys. J. 581 (2002), L93. (2002a)

27) J.-F. Liu, J. N. Bregman and P. Seitzer, Astrophys. J. 580 (2002), L31. (2002b)

28) T. J. Maccarone, A. Kundu and S. E. Zepf, Astrophys. J. 586 (2003), 814.

29) K. Makishima et al., Astrophys. J. 535 (2000), 632. 
30) A. Markowitz, R. Edelson, S. Vaughan, P. Uttley, I. M. George, R. E. Griffiths, S. Kaspi, A. Lawrence, I. McHardy, K. Nandra et al., Astrophys. J. 593 (2003), 96.

31) S. McMillan and S. Portegies Zwart, ASP Conf. Proc. 296 (2003), 85.

32) M. C. Miller and E. J. M. Colbert, astro-ph/0308402

33) F. Mirabel, Prog. Theor. Phys. Suppl. No. 155 (2004), 71.

34) R. Mushotzky, in How AGN are Found, ed. A. Barger et al. (2004).

35) S. G. Neff, J. S. Ulvestad and S. D. Campion, Astrophys. J. 599 (2003), 1043.

36) M. W. Pakull and L. Mirioni, in New Visions of the X-Ray Universe in the XMM-Newton and Chandra Era (ESA SP-488; Noordwijk: ESA, 2003), in press; astro-ph/0202488

37) D. J. Patnaude and R. A. Fesen, Astrophys. J. 587 (2003), 221.

38) T. P. Roberts and R. S. Warwick, Mon. Not. R. Astron. Soc. 315 (2000), 98.

$39)$ T. P. Roberts, M. R. Goad, M. J. Ward and R. S. Warwick, Mon. Not. R. Astron. Soc. 342 (2003), 709. (2003a)

40) T. P. Roberts and E. J. M. Colbert, Mon. Not. R. Astron. Soc. 341 (2003), L49. (2003b)

41) M. Sugiho, J. Kotoku, K. Makishima, A. Kubota, T. Mizuno, Y. Fukazawa and M. Tashiro, Astrophys. J. 561 (2001), L73.

$42)$ D. K. Strickland, E. J. M. Colbert, T. M. Heckman, K. A. Weaver, M. Dahlem and I. R. Stevens, Astrophys. J. 560 (2001), 707.

43) T. E. Strohmayer and R. F. Mushotzky, Astrophys. J. 586 (2003), L61.

$44)$ D. A. Swartz, K. K. Ghosh and A. F. Tennant, astro-ph/0302203

45) Q. D. Wang, T. Chaves and J. A. Irwin, Astrophys. J. 598 (2003), 969.

46) N. E. White, L. Stella and A. N. Parmar, Astrophys. J. 324 (1988), 363.

47) H. Wu, S. J. Xue, X. Y. Xia, Z. G. Deng and S. Mao, Astrophys. J. 576 (2002), 738.

48) M. C. Weisskopf, K. Wu, A. F. Tennant, D. A. Swartz and K. K. Ghosh, astro-ph/0311291 